\title{
Analysis of coronal magnetic fields in active regions based on detailed radio spectral data
}

\author{
S. Tokhchukova ${ }^{1} \dagger$, V. Bogod $^{2}$ \\ and V. Garaimov ${ }^{2}$ \\ ${ }^{1}$ Main (Pulkovo) Astronomical Observatory of RAS, St.Petersburg, 196140, Russia \\ email: stokh@gao.spb.ru \\ ${ }^{2}$ Special Astrophysical Observatory of RAS, Nizhnij Arkhyz, 369147, Russia \\ email: vbog@gao.spb.ru
}

\begin{abstract}
Here we present an analysis of microwave emission spectra from flare-productive active regions (FPAR). For that we used regular observations in a wide frequency range with high spectral resolution and polarization sensitivity made with the RATAN-600 radio telescope. The multi-frequency observations make possible to detect small changes of magnetic field structures at different heights of the solar atmosphere. Observations with RATAN-600 and other large radio heliographs revealed an existence of narrow-band irregularities in the circular polarization spectra of FPARs radio emission. The new receiver complex, recently installed at RATAN-600, significantly improves its technical capabilities for the FPARs further study. Now it provides $1 \%$ (or $100 \mathrm{MHz}$ ) resolution in the frequency range from $6 \mathrm{GHz}$ to $18 \mathrm{GHz}$ in simultaneous registration. We present some observational results obtained with this new equipment.
\end{abstract}

Keywords. Sun: activity, corona, chromosphere, flares, polarization, magnetic fields, radio radiation

\section{Introduction}

Radio methods provide a lot of information on coronal magnetic structures, not accessible from the X-ray or EUV wavelength observations. In particular, radio observations provide several methods of magnetic fields strength direct measurement (Gelfreikh (1999), Ryabov et al. (1999), Kundu \& Alissandrakis (1984)). However, the unique possibilities provided by radio astronomy in studying the structure and physical processes of solar active regions on the levels of upper chromosphere and the low corona, are bounded by instrumental limitations. For the reconstruction of detailed 3D structure and dynamics of active region magnetosphere, a detailed spectral and polarization analysis in a wide wavelength range, with a high polarization sensitivity is required. This could not be reached by large radio heliographs, because they provide a high spatial and temporal resolution, but could not provide relevant spectral resolution and coverage, and also they are limited in polarization sensitivity. Only two large radio telescopes RATAN-600 (Korol'kov \& Parijskij (1979)) and OVSA (Hurford (1984)), having moderate spatial resolution, completely cover the frequency range $1-18 \mathrm{GHz}$. In this range of microwave frequencies the active region's gyroresonance emission is detected. This is the main mechanism of emission during the active region evolution, up to the start of the impulse phase of a flare. The low harmonics of the range of magnetic field strengths found in the corona (up to $2500 \mathrm{G}$ ) correspond to this range of microwave frequencies. In the case of flare and preflare processes the gyrosynchrotron mechanism is possible also.

$\dagger$ Present address: Main (Pulkovo) Astronomical Observatory, 196140, St.Petersburg, Russia 

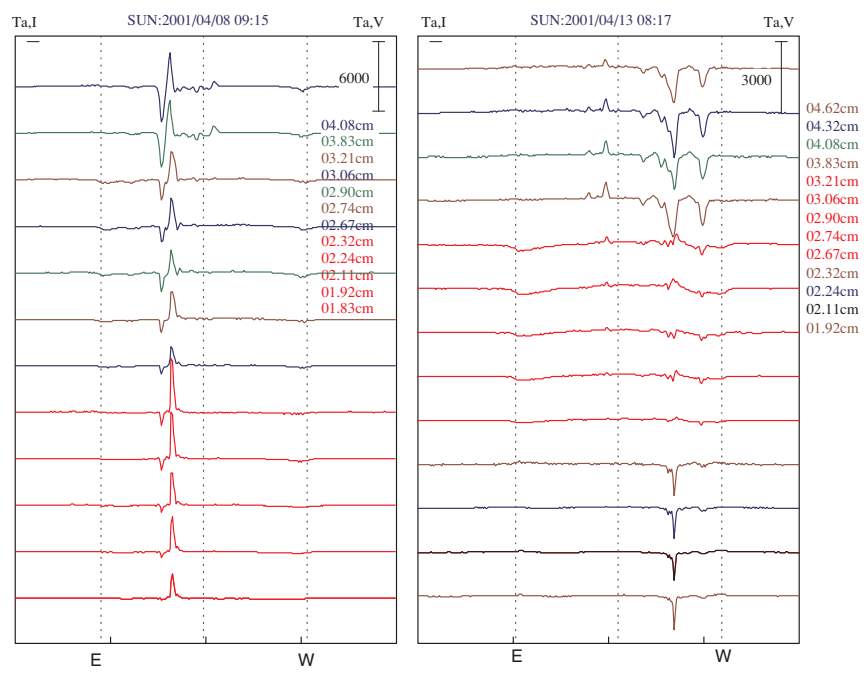

Figure 1. Left: High frequency effect of polarization brightening Right: Middle frequency effect of formation of the band of polarization depression.

\section{RATAN-600 data}

The RATAN-600 is a reflector type radio telescope with 600 meters diameter. It has the large effective area (about $1000 \mathrm{~m}^{2}$ ) and wide frequency limits (from $1 \mathrm{GHz}$ to $40 \mathrm{GHz}$ ). RATAN-600 spatial resolution (about 15 arsec at $15 \mathrm{GHz}$ ) is quite moderate compare to modern radio heliographs, but its sensitivity (fractions of s.f.u.) and polarization measurement accuracy (less than 1\%) are rather high. Another important feature of the instrument is the possibility to operate in quasi-tracking regime, providing up to $611 \mathrm{D}$ images of the Sun with 4 minutes interval. At the same time, main limitation is the difficulties to construct the frequent 2D images. Although when concerning the active region study, the approximation of circular symmetry of active regions works well. In addition, the high spectral resolution about (now about 1\%) in the range from $6 \mathrm{GHz}$ to $18 \mathrm{GHz}$ allows to receive new observational data from the low corona plasma structures.

\section{Spectral polarization features of FPARs}

Starting from the year 2000, we have analyzed a number of active regions in the sense of their spectral polarization features and their relationship with powerful flares occurrences. The statistical study revealed an existence of narrow-band irregularities in the circular polarization spectra of flare-productive active regions, in opposite to regular common spectra of stable active regions which produce no powerful flares. These active regions are characterized by a following spectral features (Bogod \& Tokhchukova (2003), Tokhchukova \& Bogod (2003)):

- increase (or reversal) of a polarization at high frequencies (within 17-10 GHz band);

- depression of polarization (up to depolarization) in a narrow frequency band (within 6-15 $\mathrm{GHz})$;

- appearance of narrow pointlike sources with multiple polarization inversions

Figure 1 demonstrates two examples of the early preflare stage manifestations, which occur only in flare productive active regions on the preflare stage of their evolution.

Interpretations for some of these these polarization peculiarities were proposed in several papers (Zlotnik (2001), Yasnov et al. (2005), Kaltman et al. (2005)) which suggest a 


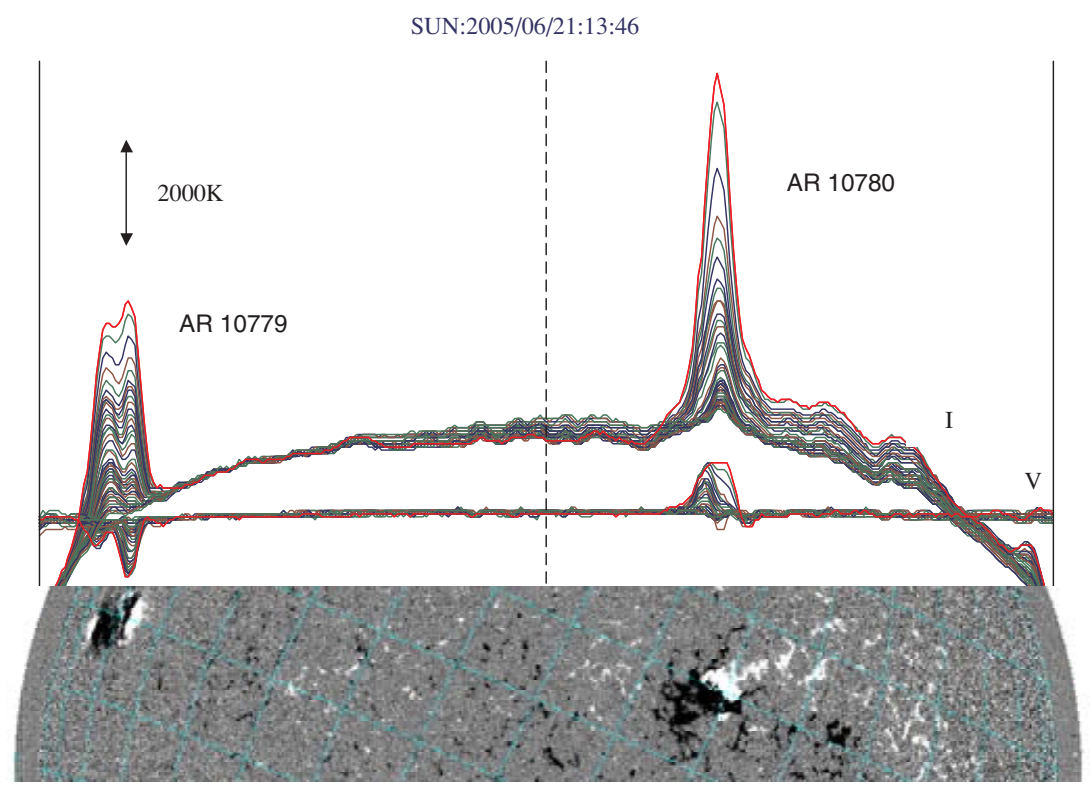

Figure 2. 1D images of the Sun with the upgraded RATAN-600 observational technique in the range 6-18 GHz. Spectral resolution between channels is 1\%. The Flare-productive AR 10780 shows a complex polarization structure on spectrum.

number of different reasons which might cause the unusual polarization behavior. For instance, the double inversion of polarization on spectrum could be explained by suggesting the existence of some layer both with temperature gradient inversion or non-homogeneous magnetic field distribution in the active region atmosphere. Detailed spectral analysis in wide frequency range is needed for the reconstruction of non-homogeneous plasma parameters (magnetic fields, electron density, temperature) profile on the height of solar atmosphere. When studying stable sunspot associated sources of radio emission, $5 \%$ spectral resolution is good enough (and necessary) to measure magnetic fields in the corona. But in the case of the newly revealed fine spectral effects, when we observe multiple inversions of polarization at adjacent frequencies, it is required more detailed analysis of spectrum. There are more tasks which require higher spectral resolution, for instance, studying the cyclotron maser emission. The new spectral-polarization receiver complex installed at RATAN-600, we hope, gives us the unique opportunities for the detailed coronal magnetography.

Figure 2 shows an example of observations with the RATAN-600 new receivers technique, at 64 wavelengths in the range from $6 \mathrm{GHz}$ to $18 \mathrm{GHz}$. Below the SOHO MDI magnetogram is provided for comparison. The structure of the AR 10780 looks quite similar at all frequencies in the channel of intensity, while in the polarization channel one can see the displacement of the emission source at several frequencies, and also the polarization inversion at high frequencies, where the emission is weaker. At this picture it is difficult to distinguish the higher frequencies because in comparison with the lower part of the spectrum their emission is much weaker. On the Figure 3 one can see the spectrum of this AR, where the polarization inversion is more evident.

Actually, now during the minimum of solar activity cycle we will try to use the new Spectrum Analyzer for study the separate active regions though the probability of strong active events is not high. 

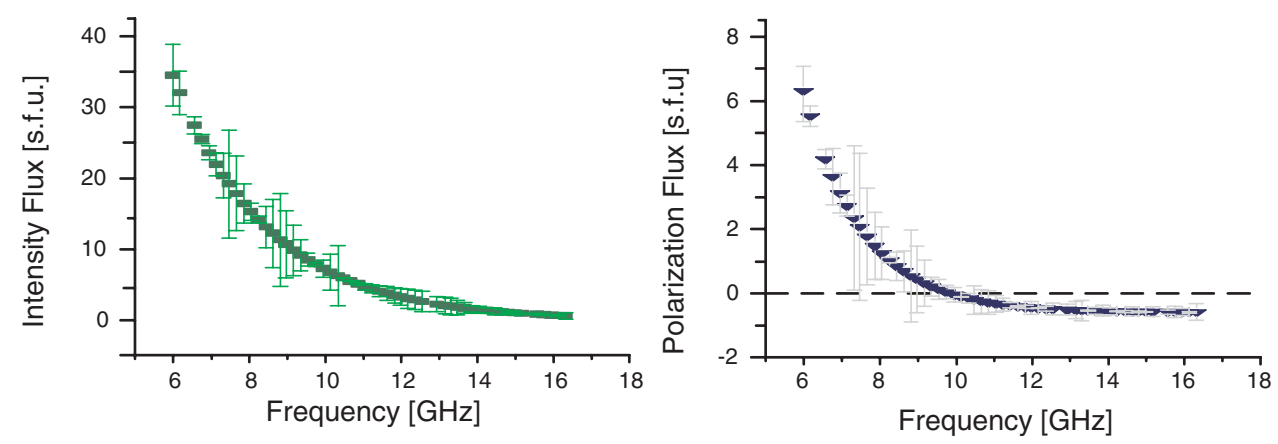

Figure 3. Intensity (left) and Polarization (right) spectra of the AR 10780. Note the polarization reversal around $10 \mathrm{GHz}$.

\section{Conclusions}

Further progress in the development and application of the observational techniques in the microwave range is a promising goal in the physics of the sun. For better understanding of physical processes, leading to big flares, the reconstruction of coronal magnetic fields strength and structure on the levels of chromosphere-low corona levels is needed. Besides a high spatial resolution and sensitivity, a fine spectral analysis technique in a wide frequency range is necessary. The new receiver complex with detailed spectralpolarization analysis recently developed and installed at RATAN-600, is a step toward to this goal.

\section{Acknowledgements}

This work is supported by RFBR under grants number 05-02-16228a and 06-02-26605.

\section{References}

Korol'kov D.V. \& Parijskij YU.N. 1979, Sky \& Telesc. 57, 324

Hurford, G.J., Read R.B. \& Zirin H. Solar Phys. 94, 413

Akhmedov Sh.B., Gelfreikh G.B, Bogod V.M. \& Korzhavin A.N. 1982, Solar Phys. 79, 41-58

Gelfreikh G.B., Bogod V.M., Abramov-Maximov V.E. \& Tsvetkov S.V. 1993, ASP Conference Series, 46, 271

Gelfreikh G.B. 1999, Solar Phys., 185, 157

Ryabov B.I., Pilyeva N.A., Alissandrakis C.E., Shibasaki K., Bogod V.M., Garaimov V.I. \& Gelfreikh G.B. 1999, Solar Phys. 85, 157-175

Kundu, M. R. \& Alissandrakis C. E. 1984, Solar Phys. 94, 249

Bogod V.M. \& Tokhchukova S.Kh. 2003, Astronomy Letters 29, N4, 263

Tokhchukova S. \& Bogod V. 2003, Solar Phys. 212, 99-109

Kaltman T.I., Korzhavin A.N. \& Y.T.Tsap 2005, Astronomy Reports 49, 9, 747-754

Zlotnik, E. 2001, Radiophysics and Quantum Electronics 44, 53-61

Yasnov, L. V., Bogod, V. M., Yan, Y. \& Kotelnikov, V. S. 2005, IAU Symposium, 223, 493-494 